\title{
The Therapy of Congenital Myasthenic Syndromes
}

\author{
Andrew G. Engel \\ Department of Neurology, Mayo Clinic, Rochester, Minnesota 55905
}

\begin{abstract}
Summary: Congenital myasthenic syndromes (CMSs) are heterogeneous disorders in which the safety margin of neuromuscular transmission is compromised by one or more mechanisms. Specific diagnosis of a CMS is important as some medications that benefit one type of CMS can be detrimental in another type. In some CMSs, strong clinical clues point to a specific diagnosis. In other CMSs, morphologic and in vitro electrophysiologic studies of the neuromuscular junction, determination of the number of acetylcholine receptors (AchRs) per junction, and molecular genetic studies may be required for a specific diagnosis. Strategies for therapy are based on whether a given CMS decreases or increases the synaptic response to
\end{abstract}

acetylcholine (ACh). Cholinesterase inhibitors that increase the synaptic response to ACh and 3,4-diaminopyridine, which increases ACh release, are useful when the synaptic response to $\mathrm{ACh}$ is attenuated. Long-lived open-channel blockers of the $\mathrm{AChR}$, quinidine, and fluoxetine, are useful when the synaptic response is increased by abnormally prolonged opening episodes of the AChR channel. Ephedrine has beneficial effects in some CMSs but its mechanism of action is not understood. Key Words: Congenital myasthenic syndromes, acetylcholine receptor, acetylcholinesterase, choline acetyltransferase, rapsyn, Dok-7, MuSK, cholinesterase inhibitors, 3,4-diaminopyridine, quinidine, fluoxetine.

\section{INTRODUCTION}

Congenital myasthenic syndromes (CMSs) represent a heterogeneous group of disorders in which the safety margin of neuromuscular transmission is compromised by one or more specific mechanisms. At the normal neuromuscular junction, activation of the postsynaptic acetylcholine receptors (AChRs) by acetylcholine (ACh) evokes an endplate potential (EPP) that activates the $\mathrm{Na}_{\mathrm{v}} 1.4$ type voltage-dependent sodium channels giving rise to a propagated action potential. A high concentration of AChRs on crests of the synaptic folds ${ }^{1}$ and of $\mathrm{Na}_{\mathrm{v}} 1.4$ in the depth of the secondary synaptic clefts ${ }^{2,3}$ ensures that excitation is propagated beyond the endplate (EP). ${ }^{4.5}$ The safety margin of neuromuscular transmission is a function of the difference between the depolarization caused by the EPP and the depolarization required to activate $\mathrm{Na}_{\mathrm{v}}$ 1.4. All CMSs identified so far have been traced to one or more factors that render the EPP subthreshold for activating $\mathrm{Na}_{\mathrm{v}} 1.4$ or to a defect in $\mathrm{Na}_{\mathrm{v}} 1.4$ itself.

The factors governing the safety margin can be grouped into the following major categories: factors that

Address correspondence and reprint requests to: Andrew G. Engel, M.D., Department of Neurology, Mayo Clinic, 200 First SW, Rochester, MN 55905; E-mail address: age@ mayo.edu. affect the number of ACh molecules per synaptic vesicle, factors that affect quantal release mechanisms, and factors that affect the efficacy of individual quanta. ${ }^{5}$ Thus, a clear understanding of the mechanisms that operate in the CMSs requires an analysis of the different ways in which these synaptic properties have been altered. They include variations in the number of AChRs per endplate, the synaptic localization of AChR and acetylcholinesterase (AChE), the fine structure of the endplate, the amplitude of miniature endplate potentials (MEPPs) and EP potentials (EPPs), the number of quanta released by nerve impulse, the number of readily releasable quanta, the probability of quantal release, and the kinetic properties of single AChRs. Combined electrophysiologic and morphologic tests can probe the involvement of these different mechanisms in CMSs. If these tests point to a candidate gene, then molecular genetic analysis becomes feasible. If a mutation is discovered in the candidate gene, then expression studies with the recombinant mutant molecule can be used to confirm its pathogenicity, analyze the electrophysiologic ${ }^{6}$ or biochemical ${ }^{7}$ properties of the mutant protein, and test drugs that may mitigate the disease. ${ }^{8-10}$ The candidate gene approach, based on clinical, morphologic, and electrophysiologic findings, has pointed to defects in choline acetyltransferase (ChAT), ${ }^{7} \mathrm{AChE},{ }^{11} \mathrm{AChR},{ }^{12}$ rapsyn, ${ }^{13}$ muscle spe- 
Table 1. Classification of Congenital Myasthenic Syndromes Based on Site of Defect*

\begin{tabular}{|c|c|}
\hline & $\begin{array}{l}\text { Index } \\
\text { cases }\end{array}$ \\
\hline \multicolumn{2}{|l|}{ Presynaptic $(8 \%)$} \\
\hline Choline acetyltransferase deficiency ${ }^{\dagger}$ & 13 \\
\hline $\begin{array}{l}\text { Paucity of synaptic vesicles and reduced } \\
\text { quantal release }\end{array}$ & 1 \\
\hline Congenital Lambert-Eaton-like & 2 \\
\hline Other presynaptic defects & 3 \\
\hline \multicolumn{2}{|l|}{ Synaptic space $(14 \%)$} \\
\hline Endplate AChE deficiency ${ }^{\dagger}$ & 35 \\
\hline \multicolumn{2}{|l|}{ Postsynaptic $(73 \%)$} \\
\hline Primary kinetic defect $\pm \mathrm{AChR}$ deficiency ${ }^{\dagger}$ & 52 \\
\hline $\begin{array}{c}\text { Primary AChR deficiency } \pm \text { minor kinetic } \\
\text { defect }^{\dagger}\end{array}$ & 92 \\
\hline Rapsyn deficiency $^{\dagger}$ & 36 \\
\hline $\mathrm{Na}^{1}$ channel myasthenia ${ }^{\dagger}$ & 1 \\
\hline Plectin deficiency & 1 \\
\hline \multicolumn{2}{|l|}{ Synaptopathy $(5 \%)$} \\
\hline Dok-7 myasthenia ${ }^{\dagger}$ & 12 \\
\hline Total & 248 \\
\hline
\end{tabular}

AChE, acetylcholinesterase; AChR, acetylcholine receptor; MuSK, muscle specific tyrosine kinase. ${ }^{*}$ Classification based on cohort of CMS patients investigated at the Mayo Clinic between 1988 and 2006. A single case of a CMS related to defect in MuSK has been reported by Chevessier et al. ${ }^{14}$ Genetic defect detected.

cific tyrosine kinase (MuSK), ${ }^{14}$ and Dok- $-7^{15}$ as causes of CMS.

\section{CLASSIFICATION OF THE CMSS}

Table 1 shows a site of defect-based classification of the CMSs and the frequency at which the different types of CMSs had been observed in the Mayo cohort of CMS patients. In this series, postsynaptic CMSs are the most frequent $(73 \%)$ and those CMS caused by a kinetic abnormality or deficiency of AChR account for $57 \%$ of the total. Mutations in rapsyn, a protein responsible for concentrating $\mathrm{AChR}$ in the postsynaptic membrane, and in ColQ, the tail subunit of AChE that anchors the enzyme in the synaptic basal lamina, each account for $\sim 14 \%$ of the cases. Each remaining syndrome accounts for $5 \%$ or fewer of the CMS cases in the Mayo cohort.

\section{EPIDEMIOLOGY}

There is no reliable information on the incidence and prevalence of CMSs. They are not uncommon, but are frequently misdiagnosed or go undiagnosed for a number of reasons: they can closely mimic other disorders (see Diagnosis and Differential Diagnosis); physicians are less familiar with CMSs than with other neurologic disorders; and the correct diagnosis of some CMSs requires specialized methods of investigation currently available only at a few medical centers. It is now known, however, that some CMSs are endemic in the Near East, where closed communities and consanguineous marriages are relatively frequent. ${ }^{16,17}$

\section{DIAGNOSIS AND DIFFERENTIAL DIAGNOSIS}

Table 2 lists clinical clues to the generic and specific diagnoses of the CMSs. However, in some patients there are no clinical clues that point to a specific type of CMS. In these patients, in vitro analysis of parameters of neuromuscular transmission, patch-clamp recordings from single AChR channels, measurement of the number of AChRs per EP, morphologic studies that include examination of the fine structure and immunocytochemical properties of the neuromuscular junction, and in some instances molecular genetic studies, are required for making a specific diagnosis. Table 3 lists the differential diagnosis of the different forms of CMSs.

\section{INHERITANCE}

In most slow-channel patients, the opening events of the AChR channel are greatly prolonged; these syndromes are caused by dominantly inherited gain-offunction mutations in different AChR subunits. Some slow-channel syndromes with less prolonged opening events of the AChR channel are determined by recessive inheritance. ${ }^{18}$ All other CMSs identified to date are caused by recessive loss-of-function mutations in various endplate-specific proteins.

\section{PRINCIPLES OF THERAPY}

Pharmacologic therapy is dictated by the defect underlying a given CMS. Drugs beneficial in one type of CMS can be harmful in another type. Therefore, specific diagnosis is essential for rational therapy.

It is important to monitor the effects of therapy by objective measures. Such measures include the arm elevation time, the number of times a patient can rise from squatting or from a low stool, the degree of eyelid ptosis, the number of steps they can climb or the distance they can walk before having to rest, the grade of weakness of selected muscles, maximal inspiratory and expiratory pressures, maximal expiratory flow, and the extent of the electromyogram decrement in one or more selected muscles.

In general terms, CMSs either decrease or increase the synaptic response to $\mathrm{ACh}$. When a CMS reduces the synaptic response, cholinesterase inhibitors, which increase the number of AChR activated by each quantum, and 3,4-diaminopyridine (3,4-DAP), which increases the 
Table 2. Diagnostic Clues in Congenital Myasthenic Syndromes (CMSs)

Generic diagnosis

- Fatigable weakness involving ocular, bulbar, and limb muscles since infancy or early childhood

- Similarly affected relative

- Decremental EMG response at 2- to 3-Hz stimulation

- Negative tests for anti-AChR antibodies, MuSK, and P/Q type calcium channels

Exceptions and caveats

- In some CMS the onset is delayed ${ }^{37,45}$

- There may be no similarly affected relatives

- The symptoms can be episodic ${ }^{7,13,44}$

- EMG abnormalities may not be present in all muscles, or are present only intermittently ${ }^{7,13}$

- Weakness may not involve cranial muscles ${ }^{40}$

Clinical clues pointing to a specific diagnosis*

Endplate AChE deficiency ${ }^{35}$

- Repetitive CMAPs

- Refractoriness to cholinesterase inhibitors

- Delayed pupillary light reflexes

Slow-channel $C M S^{45}$

- Repetitive CMAPs

- Selectively severe involvement of cervical and wrist and finger extensor muscles in most cases

- Dominant inheritance in most cases

Endplate choline acetyltransferase deficiency ${ }^{7,46}$

- Recurrent apneic episodes, spontaneous or with fever, vomiting, or excitement

- No or variable myasthenic symptoms between acute episodes

- Stimulation at $10 \mathrm{~Hz}$ for 5 min causes marked decrease of CMAP followed by slow recovery

- EMG decrement at $2 \mathrm{~Hz}$ can be absent at rest but appears after stimulation at $10 \mathrm{~Hz}$ for 5 min, then disappears slowly.

Rapsyn deficiency

- Multiple congenital joint contractures in one-fourth of patients

- Increased weakness and respiratory insufficiency precipitated by intercurrent infections

- EMG decrement can be mild or absent.

Dok-7 myasthenia ${ }^{40,15}$

- Proximal greater than distal limb and axial muscle weakness, mild facial weakness and ptosis, and normal ocular ductions in the majority

- Bulbar muscles affected in some patients

- May deteriorate on exposure to pyridostigmine

AChE, acetylcholinesterase; AChR, acetylcholine receptor; CMAP, compound muscle fiber action potential; EP, endplate; MuSK, muscle specific tyrosine kinase. *There are no clinical clues to the diagnosis of the fast channel CMS, primary EP AChR deficiency, and most cases of rapsyn deficiency.

number of quanta released by nerve impulse, are used. When the synaptic response is increased, as in the slowchannel syndromes, long-lived open-channel blockers of the AChR channel, quinidine and fluoxetine, are employed.

\section{Therapeutic agents}

Anticholinesterase medications, 3,4-DAP, quinidine, fluoxetine, and ephedrine are the mainstays of therapy in CMSs.

3,4-DAP prolongs the duration of the presynaptic action potential by blocking the outward potassium current. $^{19,20}$ This increases calcium entry into the nerve terminal when it is depolarized which, in turn, increases quantal release. The recommended dose of $3,4-\mathrm{DAP}$ is up to $1 \mathrm{mg} / \mathrm{kg} /$ day in divided doses. The drug is well tolerated, with generally only mild side effects. These include peripheral and perioral paresthesias, adrenergic side effects (palpitation, sleeplessness, ventricular extrasystoles), and cholinergic side effects (increased bronchial secretions, cough, and diarrhea). Higher doses are not recommended because of possible seizures. ${ }^{21,22}$ A previous history of seizures or potentially epileptogenic activity in the electroencephalogram are contraindications to the use of the medication. The drug is not approved for clinical use by the U.S. Food and Drug Administration, but it can be obtained as an investigational drug and used under the auspices of Institutional Review Boards. The drug is available on prescription in Canada, England, and some European countries.

The dosages and side effects of quinidine and fluoxetine are considered below, under the treatment of the slow-channel syndromes.

Ephedrine has been noted to be of some benefit in different types of CMS but its mode of action is unclear. Doses in adults range from 25 to $50 \mathrm{mg}$ two to three times daily. The dosage in children is $3 \mathrm{mg} / \mathrm{kg} /$ day in divided doses. The side effects include nervousness, insomnia, palpitation, and hypertension. 
Table 3. The Differential Diagnosis of Congenital Myasthenic Syndromes

Neonatal period, infancy, childhood

Spinal muscular atrophy

Morphologically distinct congenital myopathies (central core disease, nemaline myopathy, myotubular myopathy)

Congenital muscular dystrophies

Infantile myotonic dystrophy

Mitochondrial myopathy

Brain stem anomaly

Möbius syndrome

Congenital fibrosis of the external ocular muscles

Infantile botulism

Autoimmune myasthenia gravis*

Older patients

Motor neuron disease

Radial nerve palsy ${ }^{\dagger}$

Peripheral neuropathy ${ }^{\dagger}$

Limb girdle or facioscapulohumeral dystrophy

Mitochondrial myopathy

Chronic fatigue syndrome

AChR- or MuSK-seropositive and seronegative autoimmune myasthenia gravis

AChR, acetylcholine receptor; MuSK, muscle specific tyrosine kinase $*$ Not reported in the first year of life. ${ }^{\dagger}$ This diagnosis was suspected in some cases of the slow-channel congenital myasthenic syndromes.

\section{MANAGEMENT OF DIFFERENT TYPES OF CMSS}

\section{Endplate AChR deficiency with or without minor kinetic abnormality}

Most patients respond favorably but incompletely to cholinesterase inhibitors. The additional use of 3,4-DAP results in further significant improvement in about onethird of the cases. ${ }^{23}$ A favorable response to 3,4-DAP combined with pyridostigmine in unselected CMS patients was previously reported,${ }^{24}$ but another clinical trial of CMS patients with predominantly ocular symptoms found little response to 3,4-DAP. ${ }^{25}$ In our experience, 3,4-DAP increases endurance and reduces lid ptosis, but external ocular muscles respond less than limb muscles and in some patients the medication becomes less effective with continued use. Patients with low-expressor or null mutations in each allele of the AChR $\epsilon$ subunit can partially substitute the fetal $\gamma$ for the $\epsilon$ subunit. ${ }^{26,12}$ They usually have a milder form of CMS and respond better to therapy than patients with low-expressor mutations in both alleles of non- $\epsilon$ subunits for which there are no substituting subunits.

\section{Slow-channel syndromes}

The slow-channel syndromes are associated with slowly decaying endplate currents, destabilization of the closed channel state, prolonged activation episodes of $\mathrm{AChR}$, and an endplate myopathy caused by cationic overloading of the postsynaptic region. ${ }^{27}$ Specific ther- apy for this syndrome exists in the form of quinidine and fluoxetine. Both drugs are long-lived, open-channel blockers of AChR that shorten the duration of channel opening events in a concentration dependent manner. Five $\mu \mathrm{M} / \mathrm{L}$ of quinidine or $10 \mu \mathrm{M} / \mathrm{L}$ fluoxetine decreases the duration of slow-channel opening events severalfold to that of wild-type, but reduces wild-type channel opening events only by $\sim 50 \%$. $^{9,10}$

Adult slow-channel patients are treated with $200 \mathrm{mg}$ quinidine sulfate three times daily for one week; the dose is then gradually increased to maintain a serum level of 1 to $2.5 \mu \mathrm{g} / \mathrm{mL}$ (3-7.5 $\mu \mathrm{M} / \mathrm{L})$. After a satisfactory serum level is established, equivalent doses of a slow-release form of quinidine sulfate can be employed. The doses for children are $15-60 \mathrm{mg} / \mathrm{kg} / \mathrm{day}$ of quinidine sulfate in four to six divided doses. On this regimen, patients improve in their endurance and electromyogram tests over many months. ${ }^{28}$

Quinidine is contraindicated in CMSs other than the slow-channel syndrome. Untoward effects of quinidine include gastrointestinal reactions with diarrhea and cinchonism. Hypersensitivity to the drug can result in drug fever, abnormal liver function tests, hemolytic anemia, agranulocytosis, thrombocytopenic purpura, and toxic drug rash. Quinidine also worsens atrioventricular conduction defects, and can aggravate a prolonged QT interval, which predisposes to ventricular arrhythmias. Quinidine also inhibits cytochrome P450IIDA and thereby impairs the metabolism codeine, tricyclic antidepressants, other antiarrhythmic drugs, and digoxin. Administration of verapamil, cimetidine, and agents that alkalinize urine may augment the serum quinidine level, and quinidine potentiates the anticoagulant effects of coumadin. $^{29}$

Adult patients unable to tolerate quinidine can be treated with fluoxetine in gradually increasing doses until a total daily dose of 80 to $100 \mathrm{mg}$ is attained. ${ }^{10}$ The maximal dose of fluoxetine for children has not been established. Fluoxetine is a less effective long-lived open-channel blocker of the AChR channel than quinidine but is eliminated more slowly and therefore provides more evenly sustained blood levels. Adverse effects of fluoxetine include mild nausea, nervousness, insomnia, sexual dysfunction, and hyponatremia in the elderly. ${ }^{30}$ Fluoxetine has been reported to increase the risk of suicide-related behaviors in depressed children and adolescents. ${ }^{31,32}$ Therefore, caution is required when the medication is used in children or adolescents and it should not be used in those with signs of depression.

\section{Fast-channel syndromes}

The fast channel syndromes are associated with fast decaying endplate currents, destabilization of the open channel state, abnormally brief activation episodes of $\mathrm{AChR}$, and no anatomic footprint. ${ }^{27,33}$ Thus, they repre- 
sent a mirror image of the slow-channel syndrome. Fastchannel patients respond well to combined therapy with pyridostigmine in doses similar to those used in autoimmune MG in combination with 3,4-DAP. ${ }^{23}$ Patients without concomitant endplate AChR deficiency respond better than patients in whom the fast-channel mutation also reduces $\mathrm{AChR}$ expression.

\section{Endplate AChE deficiency}

There is no satisfactory drug therapy for this disease. Cholinesterase inhibitors cannot improve neuromuscular transmission and are contraindicated because they increase respiratory secretions and inhibit butyrylcholinesterase that partially compensates for absence of $\mathrm{AChE}$ from the EP. Some patients are subjectively and objectively improved by ephedrine sulfate; 25 to $50 \mathrm{mg}$ taken two to three times daily. ${ }^{34}$ Alternate-day prednisone therapy had a slight beneficial effect in two patients but was ineffective in one and appeared to worsen the symptoms in another. ${ }^{35}$ A severely ill respirator dependent infant was improved by intermittent blockade of AChR by atracurium, an agent that protects $\mathrm{AChR}$ from overexposure to ACh, allowing for temporary withdrawal of respiratory support. ${ }^{36}$

\section{Rapsyn deficiency}

Most patients respond well to pyridostigmine. ${ }^{37}$ Some patients derive additional benefit from the use of 3,4$\mathrm{DAP}^{38}$ or ephedrine.

\section{Choline acetyltransferase (ChAT) deficiency}

ChAT is essential for the resynthesis of ACh from choline and acetyl-coenzyme A. Mutations in ChAT impair the catalytic efficiency of the enzyme and decrease the release of ACh during physiologic activity. ${ }^{7}$ Because this CMS can cause abrupt episodes of respiratory arrest, prophylactic use of pyridostigmine is indicated once the diagnosis is established. Because apneic attacks occur suddenly in infants and children, the parents should be provided with an inflatable rescue bag and a fitted mask, they should be instructed in the intramuscular injection of neostigmine methylsulfate $(0.5 \mathrm{mg} / \mathrm{kg}$ in infants and children), and they are advised to install an apnea monitor in the home.

\section{Dok-7 myasthenia}

Dok-7 is a recently identified protein important for activation of MuSK. ${ }^{39}$ MuSK plays a role in maturation and maintenance of the synapse, and in directing rapsyn to concentrate $\mathrm{AChR}$ in the postsynaptic membrane. Mutations in Dok-7 cause progressive weakness of limbgirdle and axial muscles but only mild weakness of the facial muscles and mild eyelid ptosis. Pyridostigmine is effective in some patients, but only a few experience long-term improvement and some deteriorate even after a few days of therapy. 3,4-DAP benefited three out of eight patients and worsened one patient. Ephedrine improved five out of five patients but in one the improvement was temporary. ${ }^{40,15}$

\section{Paucity of synaptic vesicles associated with reduced quantal release}

Only one patient having this disorder has been reported. This patient responded partially to pyridostigmine. $^{41}$

\section{Lambert-Eaton syndrome-like CMS}

In the autoimmune form of the syndrome, antibodies deplete the voltage-gated calcium channel of the presynaptic membrane by antigenic modulation. This limits the ingress of calcium into the nerve terminal when it is depolarized and reduces quantal release by nerve impulse. 3,4-DAP is effective in a high proportion of patients with the autoimmune syndrome. ${ }^{42}$ One infant with the nonautoimmune congenital form of the disease observed by the author failed to respond to 3,4-DAP.

\section{CMS caused by defect in MuSK}

In a single reported patient pyridostigmine was ineffective but 3,4-diaminopyride had a beneficial effect. ${ }^{14}$

\section{CMS caused by plectin deficiency}

Plectin is an intermediate filament-associated protein concentrated at sites of mechanical stress. Pathogenic mutations in plectin are associated with a simplex variety of epidermolysis bullosa, and, in some cases, with a progressive myopathy and a defect of neuromuscular transmission. One patient with this type of myasthenia failed to respond to pyridostigmine but was improved by 3,4-DAP. ${ }^{43}$

\section{Na channel myasthenia}

In a single reported patient, therapy with pyridostigmine improved the patient's endurance; additional therapy with acetazolamide, which is known to mitigate periodic paralysis due to $\mathrm{Na}_{\mathrm{v}} 1.4$ mutations, prevented further attacks of respiratory and bulbar weakness. ${ }^{44}$

\section{General measures}

Severely affected CMS patients are born with or develop a restrictive ventilatory defect, cannot swallow, and develop progressive spinal deformities. Some CMS infants are unable to breathe at birth but can be weaned off the respirator after a few months. Ventilatory defects developing in later life initially require nocturnal and later daytime ventilatory support. Spinal deformities must be carefully monitored; if progressive and significant, corrective surgery is indicated. Surgery is best done in the early teens, after vertebral growth has ceased. Severe dysphagia requires feeding via a gastrostomy tube. With early specific diagnosis and therapy, the above life-threatening consequences of the CMSs can be mitigated or obviated. 


\section{REFERENCES}

1. Salpeter MM. Vertebrate neuromuscular junctions: general morphology, molecular organization, and functional consequences. In: Salpeter MM, ed. The vertebrate neuromuscular junction. New York: Wiley; 1987:1-54.

2. Flucher BE, Daniels MP. Distribution of $\mathrm{Na}+$ channels and ankyrin in neuromuscular junctions is complementary to that of acetylcholine receptors and the $43 \mathrm{kd}$ protein. Neuron 1989;3:163-175.

3. Ruff RL. Sodium channel slow inactivation and the distribution of sodium channels on skeletal muscle fibres enable the performance properties of different skeletal muscle fiber types. Acta Physiol Scand 1996;156:159-168.

4. Martin AR. Amplification of neuromuscular transmission by postjunctional folds. Proc R Soc Lond B Biol Sci 1994;258:321-326.

5. Wood SJ, Slater CP. Safety factor at the neuromuscular junction. Prog Neurobiol 2001;64:393-429.

6. Engel AG, Ohno K, Milone M, et al. New mutations in acetylcholine receptor subunit genes reveal heterogeneity in the slowchannel congenital myasthenic syndrome. Hum Mol Genet 1996; 5:1217-1227.

7. Ohno K, Tsujino A, Brengman JM, et al. Choline acetyltransferase mutations cause myasthenic syndrome associated with episodic apnea in humans. Proc Natl Acad Sci U S A 2001;98:2017-2022.

8. Engel AG. The investigation of congenital myasthenic syndromes. Ann NY Acad Sci 1993;681:425-434.

9. Fukudome T, Ohno K, Brengman JM, Engel AG. Quinidine normalizes the open duration of slow-channel mutants of the acetylcholine receptor. Neuroreport 1998;9:1907-1911.

10. Harper CM, Fukudome T, Engel AG. Treatment of slow channel congenital myasthenic syndrome with fluoxetine. Neurology 2003; 60:170-173.

11. Ohno K, Brengman JM, Tsujino A, Engel AG. Human endplate acetylcholinesterase deficiency caused by mutations in the collagen-like tail subunit (ColQ) of the asymmetric enzyme. Proc Natl Acad Sci U S A 1998;95:9654-9659.

12. Ohno K, Quiram PA, Milone M, et al. Congenital myasthenic syndromes due to heteroallelic nonsense/missense mutations in the acetylcholine receptor e subunit gene: identification and functional characterization of six new mutations. Hum Mol Genet 1997;6: 753-766.

13. Ohno K, Engel AG, Shen XM, et al. Rapsyn mutations in humans cause endplate acetylcholine receptor deficiency and myasthenic syndrome. Am J Hum Genet 2002;70:875-885.

14. Chevessier F, Faraut B, Ravel-Chapuis A, et al. MUSK, a new target for mutations causing congenital myasthenic syndrome. Hum Mol Genet 2004;13:3229-3240.

15. Beeson D, Higuchi O, Palace J, et al. Dok-7 mutations underlie a neuromuscular junction synaptopathy. Science 2006;313:1975-1978.

16. Ohno K, Anlar B, Ozdirim E, Brengman JM, DeBleecker JL, Engel AG. Myasthenic syndromes in Turkish kinships due to mutations in the acetylcholine receptor. Ann Neurol 1998;44:234241.

17. Middleton L, Ohno K, Christodoulou K, Sine SM. Congenital myasthenic syndromes linked to chromosome $17 \mathrm{p}$ are caused by defects in acetylcholine receptor e subunit gene. Neurology 1999; 53:1076-1082.

18. Croxen R, Hatton C, Shelley C, et al. Recessive inheritance and variable penetrance of slow-channel congenital myasthenic syndromes. Neurology 2002;59:162-168.

19. Maeno T. Kinetic analysis of a large facilitatory action of 4-aminopyridine on the motor nerve terminal of the neuromuscular junction. Proc Jpn Acad 1980;56:241-245.

20. Saint DA. The effects of 4-aminopyridine and tetraethylammonium on the kinetics of transmitter release at the mammalian neuromuscular synapse. Can J Physiol Pharmacol 1989;67:10451050.

21. McEvoy KM, Windebank AJ, Daube JR, Low PA. 3,4-Diaminopyridine in the treatment of Lambert-Eaton myasthenic syndrome. N Engl J Med 1989;321:1567-1571.

22. Sanders DB, Howard JF Jr, Massey JM. 3,4-diaminopyridine in Lambert-Eaton myasthenic syndrome and myasthenia gravis. Ann NY Acad Sci 1993;681:588-590.
23. Harper CM, Engel AG. Treatment of 31 congenital myasthenic syndrome patients with 3,4-diaminopyridine. Neurology 2000; 54(suppl 3):A395.

24. Palace J, Wiles CM, Newsom-Davis J. 3,4-diaminopyridine in the treatment of congenital (hereditary) myasthenia. J Neurol Neurosurg Psychiatry 1991;54:1069-1072.

25. Anlar B, Varli K, Ozdirim E, Ertan M. 3,4-diaminopyridine in childhood myasthenia: double blind, placebo controlled trial. J Child Neurol 1996;11:458-461.

26. Engel AG, Ohno K, Bouzat C, Sine SM, Griggs RG. End-plate acetylcholine receptor deficiency due to nonsense mutations in the e subunit. Ann Neurol 1996;40:810-817.

27. Engel AG, Ohno K, Sine SM. Sleuthing molecular targets for neurological diseases at the neuromuscular junction. Nat Rev Neurosci 2003;4:339-352.

28. Harper CM, Engel AG. Quinidine sulfate therapy for the slowchannel congenital myasthenic syndrome. Ann Neurol 1998;43: $480-484$

29. Drug Evaluations Annual, 7th ed. Milwaukee, WI: American Medical Association; 1995:677-679.

30. Drug Evaluations Annual, 7th ed. Milwaukee, WI: American Medical Association; 1995:310-311.

31. Whittington CJ, Kendall T, Pilling S. Are the SSRIs and atypical antidepressants safe and effective for children and adolescents? Curr Opin Psychiatry 2005;18:21-25.

32. Bailly D. Efficacy of selective serotonin reuptake inhibitor treatment in children and adolescents. Presse Med 2006;35:1293-1302.

33. Engel AG, Ohno K, Sine SM. Congenital myasthenic syndromes. In: Engel AG, ed. Myasthenia gravis and myasthenic disorders. New York: Oxford University Press; 1999:251-297.

34. Bestue-Cardiel M, Saenz de Cabezon-Alvarez A, Capablo-Liesa $\mathrm{JL}$, et al. Congenital endplate acetylcholinesterase deficiency responsive to ephedrine. Neurology 2005;65:144-146.

35. Hutchinson DO, Walls TJ, Nakano S, et al. Congenital endplate acetylcholinesterase deficiency. Brain 1993;116:633-653.

36. Breningstall GN, Kurachek SC, Fugate JH, Engel AG. Treatment of congenital endplate acetylcholinesterase deficiency by neuromuscular blockade. J Child Neurol 1996;11:345-346.

37. Burke G, Cossins J, Maxwell S, et al. Rapsyn mutations in hereditary myasthenia: distinct early- and late-onset phenotypes. Neurology 2003;61:826-828.

38. Banwell BL, Ohno K, Sieb JP, Engel AG. Novel truncating RAPSN mutation causing congenital myasthenic syndrome responsive to 3,4-diaminopyridine. Neuromuscul Disord 2004;14: 202-207.

39. Okada K, Inoue A, Okada M, et al. The muscle protein Dok-7 is essential for neuromuscular synaptogenesis. Science 2006;312:18021805.

40. Slater CR, Fawcett PRW, Walls TJ, et al. Pre- and postsynaptic abnormalities associated with impaired neuromuscular transmission in a group of patients with 'limb-girdle myasthenia'. Brain 2006;129:2061-2076.

41. Walls TJ, Engel AG, Nagel AS, Harper CM, Trastek VF. Congenital myasthenic syndrome associated with paucity of synaptic vesicles and reduced quantal release. Ann N Y Acad Sci 1993;681: 461-468.

42. Tim RW, Massey JM, Sanders DB. Lambert-Eaton myasthenic syndrome: electrodiagnostic findings and response to treatment. Neurology 2000;54:2176-2178.

43. Banwell BL, Russel J, Fukudome T, Shen XM, Stilling G, Engel AG. Myopathy, myasthenic syndrome, and epidermolysis bullosa simplex due to plectin deficiency. J Neuropathol Exp Neurol 1999; $58: 832-846$

44. Tsujino A, Maertens C, Ohno K, et al. Myasthenic syndrome caused by mutation of the SCN4A sodium channel. Proc Natl Acad Sci U S A 2003;100:7377-7382.

45. Engel AG, Lambert EH, Mulder DM, et al. A newly recognized congenital myasthenic syndrome attributed to a prolonged open time of the acetylcholine-induced ion channel. Ann Neurol 1982; 11:553-569.

46. Byring RF, Pihko H, Tsujino A, et al. Congenital myasthenic syndrome associated with episodic apnea and sudden infant death. Neuromuscul Disord 2002;12:548-553. 